\title{
Rapid Voxel Classification Methodology for Interactive 3D Medical Image Visualization
}

\author{
Qi Zhang, Roy Eagleson, and Terry M. Peters \\ Imaging Research Laboratories, Robarts Research Institute, \\ Biomedical Engineering, University of Western Ontario, \\ London, Ontario, N6A 5K8, Canada \\ \{qzhang, eagleson, tpeters\}@imaging.robarts.ca
}

\begin{abstract}
In many medical imaging scenarios, real-time high-quality anatomical data visualization and interaction is important to the physician for meaningful diagnosis 3D medical data and get timely feedback. Unfortunately, it is still difficult to achieve an optimized balance between real-time artifact-free medical image volume rendering and interactive data classification. In this paper, we present a new segment-based post color-attenuated classification algorithm to address this problem. In addition, we apply an efficient numerical integration computation technique and take advantage of the symmetric storage format of the color lookup table generation matrix. When implemented within our GPUbased volume raycasting system, the new classification technique is about 100 times faster than the unaccelerated pre-integrated classification approach, while achieving the similar or even superior quality volume rendered image. In addition, we propose an objective measure of artifacts in rendered medical image based on high-frequency spatial image content.
\end{abstract}

\section{Introduction}

The dramatically increased capabilities of computerized radiology equipment, such as CT or MRI, have made 3D and 4D (space and time) medical images ubiquitous in surgical planning, diagnosis and therapy. Direct volume rendering has proven to be an effective and flexible method for clinical dataset visualization [1. However, in practice, the lack of an efficient strategy to map the scalar voxel value to the appropriate optical properties limits its wide applications in medicine [2].

The transfer function (TF) provides the mapping from scalar values to emitted radiant colors and extinction coefficients, thus rendering the scalar data visible and can isolate specific features in the rendered medical data. According to the sampling theorem, a continuous signal can be correctly reconstructed from its values at discrete sampling points with a sampling rate higher than the Nyquist frequency. The TF can either be applied directly to the discrete voxel points before the data interpolation step, or alternatively to the sampling points derived from the interpolation of the nearby voxel points, i.e., after the data interpolation. These processes are called pre- and post-classification respectively 3. However, both algorithms introduce high spatial frequency artifacts 


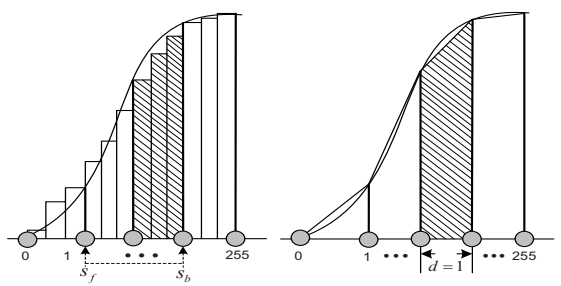

(a)

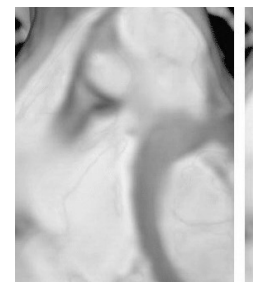

(b)

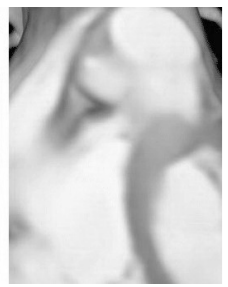

(c)

Fig. 1. The schematic of two numerical integration computation methods and the corresponding volume rendered clipped cardiac images: (a) two numerical integration calculation approaches with one marked approximating step; (b) volume rendered image with undersampling artifacts whose pre-integrated classification LUT is approximated using the middle Riemann sum with $k$ set to 8 ; (c) artifact-free volume rendered image whose post color-attenuated classification LUT is approximated using the trapezoidal rule based recursive formula with the sampling step set to 1

into the original signal. Pre-classification (PreC) simply cuts off the high frequencies, making the rendered image fuzzy, while the post-classification (PosC) takes them into account, resulting in observable undersampling artifacts in the final images. To address this problem, Röttger et al. 44 and Engel et al. 5] proposed a pre-integrated classification (PIC) algorithm, which renders the volume slab-by-slab, instead of slice-by-slice. Because of the high computational cost in the color lookup table (LUT) generation, Engel et al. also proposed a hardware acceleration technique for a texture-mapping based volume rendering [5]. However, this dependent texture based acceleration approach is not suitable for our raycasting system that is implemented on a graphics processing unit (GPU) 6], which usually produces superior images to those generated by texture mapping. Moreland and Angel [7] presented a partial PIC method to accelerate the unstructured data classification. However, this algorithm needs to load a very large integration table into the graphics memory during the raycasting process, which seriously degrades the rendering efficiency.

\section{Method}

\subsection{Pre- and Post-classification}

In our application, the classification is implemented through a TF editor, which creates a LUT. The editor is designed graphically, allowing the user to interact with the four one-dimensional piecewise curves with the range $[0, L](L=255)$ in the $x$-axis representing the scalar values, and $[0, L]$ in the $y$-axis describing the mapped optical properties.

In the process of PreC, each scalar value in the $3 \mathrm{D}$ volume is first cast to the range $[0, L]$, and then mapped to the color and opacity by the LUT. Next, the classified data are loaded into the GPU fragment shader as a 3D texture, which is utilized in the raycasting computation. In the PosC application, the generated 
LUT is first loaded into the GPU as a 1D texture, and then the volumetric data are transformed to texture space and also loaded into the GPU as a 3D texture with a single alpha component storing the scalar value. At each sampling point along the casting ray on the fragment shader, a 3D texture lookup is employed to fetch the interpolated scalar value, and then a 1D texture lookup is applied to map it to color and opacity.

\subsection{Pre-integrated Classification}

In order to form an objective comparison to our new algorithm, we have characterized a generic pre-integrated classification method as follows. For two integers $s_{f}$ and $s_{b}\left(s_{f}, s_{b} \in[0, L]\right)$ on the $x$-axis of the TF editor, we calculate the integrated opacity associated color and opacity using Eqs. (11) and (2), where $c(x)$ $\left(x \in\left[s_{f}, s_{b}\right]\right)$ is the non-associated color derived from the TF, and $\tau(x)$ is the corresponding extinction coefficient. All the possible combinations of $s_{f}$ and $s_{b}$ are calculated, resulting in a 2D LUT. As shown in the left part of Fig 1 (a), the middle Riemann sum is used to approximate the integrals expressed by Eqs. (1) and (2), and the sampling step $d$ is set to $\left|s_{b}-s_{a}\right| / n$, where $n=k+\left|s_{b}-s_{a}\right|$. Based on our experiment, $k$ should be usually chosen to be larger than 15 to ensure sufficient accuracy, and to avoid the undersampling artifacts, as shown in Fig[1(b).

$$
\begin{aligned}
\alpha\left(s_{f}, s_{b}\right) \approx & 1-\exp \left(-\int_{0}^{1} \tau\left((1-\zeta) s_{f}+\zeta s_{b}\right) d \zeta\right) \\
\tilde{C}\left(s_{f}, s_{b}\right) \approx & \int_{0}^{1} \tau\left((1-\zeta) s_{f}+\zeta s_{b}\right) c\left((1-\zeta) s_{f}+\zeta s_{b}\right) \\
& \times \exp \left(-\int_{0}^{\zeta} \tau\left(\left(1-\zeta^{\prime}\right) s_{f}+\zeta^{\prime} s_{b}\right) d \zeta^{\prime}\right) d \zeta
\end{aligned}
$$

The generated LUT is then loaded into the GPU fragment shader as a 2D texture for classification. Because no high frequency components are introduced by the nonlinear TF, the PIC has fewer undersampling artifacts and higher image quality than the PreC and PosC algorithms. However, since we need to reconstruct the LUT whenever the TF changes, it is difficult to perform classification interactively.

\subsection{Post Color-Attenuated Classification}

Considering the noninteractivity in the pre-integrated LUT generation, we propose a new post color attenuated classification (PCAC) algorithm to evaluate the segment optical properties rapidly. We first calculate the non-associated color integration for integers $s_{f}$ and $s_{b}$ on the $x$-axis of the TF editor using Eq.(3), where $c(x)$ is the non-associated color as described in subsection 2.2

$$
C\left(s_{f}, s_{b}\right) \approx \int_{0}^{1} c\left((1-\zeta) s_{f}+\zeta s_{b}\right) d \zeta=\frac{1}{s_{b}-s_{f}}\left(\int_{0}^{s_{b}} c(\omega) d \omega-\int_{0}^{s_{f}} c(\omega) d \omega\right)
$$



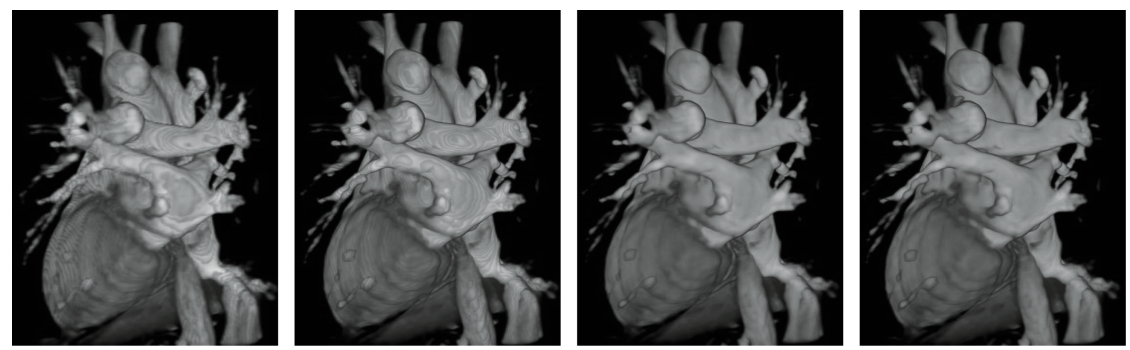

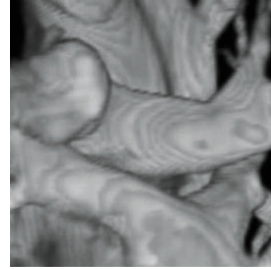

(a: pre-class.)

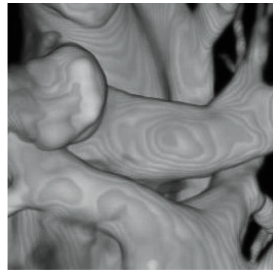

(b: post-class.)

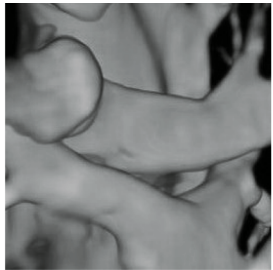

(c: pre-inte.)

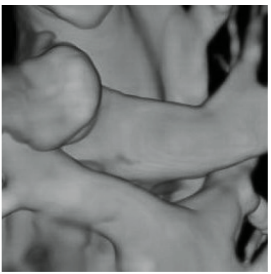

(d: pos. col. att.)

Fig. 2. Image quality comparisons of four volume classification algorithms. The visualization engine is a raycasting volume renderer implemented on a GPU, and the data is a $3 \mathrm{D}$ cardiac MR image.

Next we calculate the corresponding opacity using an alternative form of $\alpha$ :

$$
\alpha\left(s_{f}, s_{b}\right) \approx 1-\exp \left[-\frac{1}{s_{b}-s_{f}}\left(\int_{0}^{s_{b}} \tau(\omega) d \omega-\int_{0}^{s_{f}} \tau(\omega) d \omega\right)\right]
$$

Using the procedures described in the following subsection 2.4 and 2.5 we can build a 2D LUT from Eqs. (3) and (4), and then load it into the GPU fragment shader as a 2D texture. During the raycasting process on the fragment shader, for every two consecutive sampling points $\mathbf{x}(i)$ and $\mathbf{x}(i+1)$ on the casting ray $\mathbf{x}(t)$, first fetch the scalar values $s_{f}=s(\mathbf{x}(i))$ and $s_{b}=s(\mathbf{x}(i+1))$ through $3 \mathrm{D}$ texture lookup, which are then utilized as texture coordinates to address the $2 \mathrm{D}$ LUT to get the non-associated color $C_{i}=C\left(s_{f}, s_{b}\right)$ and opacity $\alpha_{i}$ $=\alpha\left(s_{f}, s_{b}\right)$ of the segment $\mathbf{x}(i) \mathbf{x}(i+1)$. According to the model of optical light emission and absorption, when colored light passes through a segment, its intensity is attenuated because of the segment's opacity. Now on the casting ray, we compute the color intensity attenuation segment by segment. Therefore, the opacity associated color of the segment $\mathbf{x}(i) \mathbf{x}(i+1)$ is calculated by the formula $\tilde{C}_{i}=\tilde{C}\left(s_{f}, s_{b}\right)=\alpha_{i}\left(s_{f}, s_{b}\right) C_{i}\left(s_{f}, s_{b}\right)$, and the volume rendering equation $\sum_{i=0}^{n} \tilde{C}_{i} \prod_{j=0}^{i-1}\left(1-\alpha_{j}\right)$ is utilized to compute the final rendered pixel. Because we calculate the color attenuation of the whole ray segment after determining the color accumulation within it, we refer to our algorithm as segment-based post color-attenuated classification.

Our PCAC algorithm is different from the accelerating method proposed in [5], which uses $c(\omega) \tau(\omega)$ to submit the $c(\omega)$ in Eq. (3) to calculate the opacity associated color $\tilde{C}\left(s_{f}, s_{b}\right)$ of the segment $\mathbf{x}(i) \mathbf{x}(i+1)$, or directly employs the 
voxel's associated color $\tilde{c}(\omega)$ to calculate the segment's opacity associated color $\tilde{C}\left(s_{f}, s_{b}\right)$. Here, the $c(\omega)$ is the non-associated color and $\tau(\omega)$ is its extinction coefficient, which are derived from the scalar value $\omega\left(\omega \in\left[s_{f}, s_{b}\right]\right)$ through the TF. This algorithm therefore computes the color attenuation voxel by voxel within the ray segment. However, this computed voxel-based associated color of the segment $\mathbf{x}(i) \mathbf{x}(i+1)$ cannot produce a correct rendering result when it is used in the above described volume rendering equation.

\subsection{Efficient Numerical Integration Computation}

From subsection 2.2, we note that the most time-consuming part in the preintegrated LUT generation is the numerical integration calculation, since for every different segment, its sampling step is changed. Therefore, when we compute a new integration, the previous results cannot be used. As demonstrated by Fig. 1(a), when estimating a nonlinear TF, a piecewise linear function is usually more accurate than a piecewise constant function. Here, we propose a recursive formula for efficient and accurate calculation of the numerical integrals. Each channel of our TF can be considered as a continuous function $f(x)$ passing through the evenly separated points $f(0), f(1), \cdots, f(L)$. We use the trapezoidal rule, a linear approximation of the Simpson's rule, to approximately calculate the definite integral from $i$ to $i+1(i=0,1, \cdots, L-1)$.

$$
\int_{i}^{i+1} f(x) d x \approx \frac{1}{6}\left[f(i)+4 f\left(i+\frac{1}{2}\right)+f(i+1)\right] \approx \frac{1}{2}[f(i)+f(i+1)]
$$

When $\left|f^{\prime \prime}(x)\right| \leq K$ for all $x \in[i, i+1]$, the approximating error is less than $K / 12$. Since each channel our TF is designed to be smooth between every two consecutive sampling points, we can therefore ensure that the $K$ is sufficiently small. The integral $\Phi(N)=\int_{0}^{N} f(x) d x(N=0,1, \cdots L)$ can then be evaluated with the following recursive formula:

$$
\Phi(N) \approx \frac{1}{2} \sum_{i=0}^{N}[f(i)+f(i+1)]=\Phi(N-1)+\frac{1}{2}[f(N)+f(N+1)]
$$

Here, $f(L+1)=0$, and when $N=0, \Phi(N-1)$ is equal to zero. We can see that in our integral calculation, only $L+1$ calculations are needed to get all the integrals required by Eqs.(3) and (4) for the LUT generation.

\subsection{Symmetric Matrix Filling}

Since the two endpoints of every segment are symmetric, we only need to compute the numerical integrals of $C\left(s_{f}, s_{b}\right)$ and $\alpha\left(s_{f}, s_{b}\right)$ with $s_{f} \leq s_{b}$ using Eqs.(3) and (4), in which the color and opacity integrals $\int_{0}^{s} c(x) d x$ and $\int_{0}^{s} \tau(x) d x$ are calculated using Eq.(6). This approach creates a symmetric matrix $M_{i, j, k}(i, j=$ $0, \cdots, L, k=0, \cdots, 3)$ for color and opacity mapping, and we fill the entries $M(i, j, k)$ and $M(j, i, k)$ synchronously with the same value, reducing the matrix generation time. This matrix is then loaded into the GPU fragment shader as a 2D texture LUT for scalar value classification. 
Table 1. Performance comparisons of volume rendering (Rend. - frames per second) and classification (Clas. - millisecond per classification) using five medical datasets.

\begin{tabular}{c|c|c|c|c|c|c|c|c|c|c}
\hline Vol. & Dataset & Voxel Size & \multicolumn{2}{|c|}{ Pre-Class. } & \multicolumn{2}{|c|}{ Post-Class. } & \multicolumn{2}{|c|}{ Pre-Integ. } & \multicolumn{2}{l}{ P. Col. Att. } \\
\cline { 5 - 10 } No. & Dimension & $(\mathrm{mm})$ & Rend. & Clas. & Rend. & Clas. & Rend. & Clas. & Rend. & Clas. \\
\hline \hline $\mathrm{D}_{1}$ & $(115,170,75)$ & $(1.48,1.48,1.50)$ & 54.2 & 60 & 50.0 & 0.05 & 54.8 & 420 & 55.5 & 4.1 \\
$\mathrm{D}_{2}$ & $(160,144,208)$ & $(0.12,0.12,0.08)$ & 17.3 & 132 & 16.3 & 0.05 & 18.1 & 420 & 17.9 & 4.1 \\
$\mathrm{D}_{3}$ & $(200,200,281)$ & $(1.00,1.00,1.00)$ & 38.2 & 280 & 37.1 & 0.05 & 39.1 & 420 & 39.5 & 4.1 \\
$\mathrm{D}_{4}$ & $(181,217,181)$ & $(1.00,1.00,1.00)$ & 26.8 & 195 & 25.0 & 0.05 & 28.4 & 420 & 28.6 & 4.1 \\
$\mathrm{D}_{5}$ & $(394,394,181)$ & $(0.50,0.50,0.50)$ & 43.0 & 685 & 49.5 & 0.05 & 54.2 & 420 & 54.5 & 4.1 \\
\hline
\end{tabular}

\section{$3 \quad$ Experimental Results}

We have implemented the new classification algorithm in our medical image visualization system that comprises a Pentium IV 3.2GHz CPU, an Nvidia GeForce 7900 GTO GPU, and 2GB main memory, using C++, OpenGL, OpenGL Shading Language (GLSL) and Qt.

\subsection{Performance Comparisons}

We employed five datasets from three different imaging modalities to test the algorithm performance (Table 1). $\mathrm{D}_{1}$ is a MR volume of human heart (Fig. 2), $\mathrm{D}_{2}$ is a cardiac 3D US of the same subject as $\mathrm{D}_{1}, \mathrm{D}_{3}$ is a CT of a human heart within the thorax, $\mathrm{D}_{4}$ is a CT brain and $\mathrm{D}_{5}$ is a CT of a pig heart (Fig. 33). As illustrated in Table 1, our PCAC algorithm takes $4.1 \mathrm{~ms}$ for per volume classification, about 100 times faster than the PIC method. The PosC takes the shortest time, since it only needs to calculate a 1D LUT and transfer it to the GPU as a 1D texture. Unlike the other algorithms, which take the same time to classify different dataset, the PreC requires a classification time that is almost directly proportional to the volume size, since it needs to classify each voxel and update the whole volume to the GPU.

Table 1 also compares the performance of different classification algorithms on the same volume renderer, from which we note that our PCAC algorithm has almost the same efficiency as the PIC, and about 10\% faster than the PreC and PosC. Usually the average speed difference is less than $15 \%$ of the four algorithms, depending on the rendered dataset. We believe that this is because some classifications map the same scalar value to a higher opacity than the others, and the application of the early ray termination in our system causes the casting rays to terminate earlier in higher opacity volumes than those in lower opacities.

\subsection{Image Quality Evaluation}

We evaluate the quality of the rendered images both subjectively and objectively. The image qualities are compared in an image-guided cardiac surgery planning 

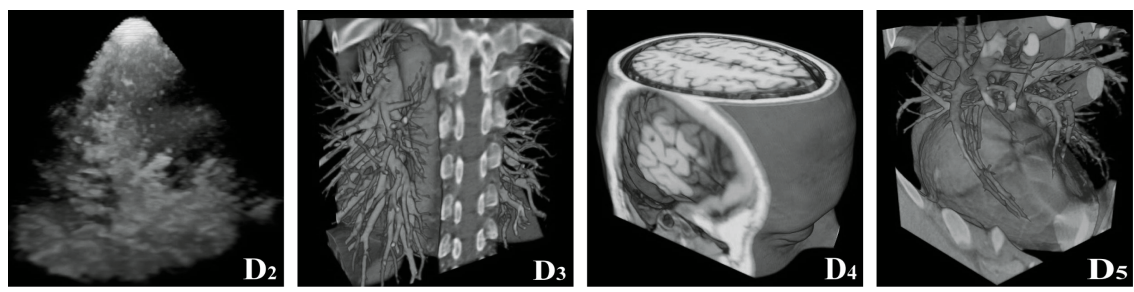

Fig. 3. Volumetric medical images rendered with the GPU-based raycasting system, utilizing the post color-attenuated classification algorithm
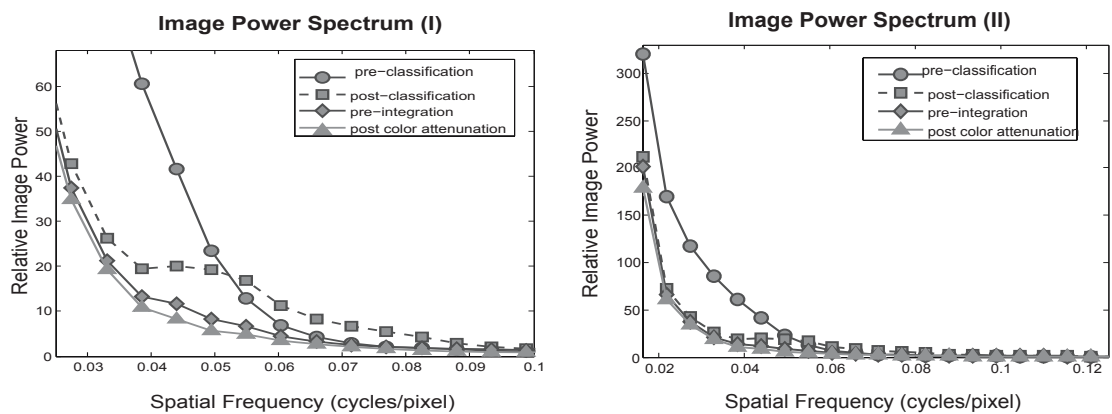

Fig. 4. The radial plots noise power spectrums of four sets (20 images) of images: (I) specific frequency range description; (II) large frequency range illustration

process, during which one experienced cardiologist and two cardiac imaging specialists interactively adjust the transfer function to capture the target anatomical structure separately. As shown in Fig. 2, they give the images in the column (c) and (d) the highest rank based on the facility, clarity and accuracy of the target structure navigation, while rating the images in the column (a) the lowest.

To quantitatively assess the visual performance, we employed noise power spectrum (NPS) analysis 8 to quantify the artifacts. Using a standard sphere as a test object, we acquired four image sets, each generated with different classification algorithms and including twenty random views of the volume-rendered image. We then computed the magnitude of the mean image power spectrum (IPS) of each image set. Since the structure in the ideal image is contained mainly in the low spatial frequency $(\mathrm{SF})$ components $(<0.01$ cycles/pixel), components in the higher frequency region reveal the SF of the introduced artifacts. In Fig. 4 , the $2 \mathrm{D}$ spectral images have been reduced to radial plots of the average IPS in an annulus at specific SF (distance) from the center (zero frequency). When the $\mathrm{SF}$ is lower than 0.052 cycles/pixel, the PreC has the highest noise power, while the PosC has the second highest. When the SF is higher than this threshold, the PosC has the highest artifact energy. Throughout the entire SF range, our classification algorithm and the PIC have similar low noise performance, while our algorithm generated $\sim 10 \%$ lower artifact levels than the PIC. Therefore, when applied in the same raycasting based medical image volume rendering system, 
the PreC introduces significant low frequency noise, while the PosC creates high frequency noise, and our PCAC algorithm introduces the lowest noise along the entire frequency range, producing the highest rendered image quality.

\section{Conclusions}

In this paper, we present a new post color-attenuated volume classification algorithm, which calculates the color attenuation on a segment basis, and has been implemented in our GPU-based medical image real-time 3D visualization system for interactive volume classification. We also introduce an efficient numerical integration approximation technique to accelerate the LUT generation, during which the symmetric data storage format of the optical mapping matrix is utilized. Besides high rendered image quality, our algorithm takes approximately 4 milliseconds, about 100 times faster than an unaccelerated pre-integrated classification approach, making this technique compatible with screen rate realtime constants. We believe our new interactive classification and real-time volume rendering techniques will facilitate the interactive visualization of the medical image datasets in both diagnostic and therapeutic applications.

\section{References}

1. Bühler, K., Neubauer, A., Hadwiger, M., Wolsfberger, S., Wegenkittl, R.: Interactive 3D techniques for computer aided diagnosis and surgery simulation tools. In: Hruby, W. (ed.) Digital Revolution in Radiology - Bridging the Future of Health Care, 2nd edn., Springer, Heidelberg (2005)

2. Kniss, J., Kindlmann, G., Hansen, C.: Multidimensional transfer functions for interactive volume rendering. IEEE Transactions on Visualization and Computer Graphics 8(3), 270-285 (2002)

3. Rezk-Salama, C.: Volume Rendering Techniques for General Purpose Graphics Hardware. PhD thesis, University of Siegen, Germany (2001)

4. Röttger, S., Kraus, M., Ertl, T.: Hardware-accelerated volume and isosurface rendering based on cell-projection. In: Proceedings of IEEE Visualization 2000, pp. 109-116. IEEE Computer Society Press, Los Alamitos (2000)

5. Engel, K., Kraus, M., Ertl, T.: High-quality pre-integrated volume rendering using hardware-accelerated pixel shading. In: HWWS 2001. Proceedings of the ACM SIGGRAPH/EUROGRAPHICS workshop on Graphics hardware, pp. 9-16. ACM Press, New York, NY, USA (2001)

6. Zhang, Q., Eagleson, R., Peters, T.M.: Real-time visualization of 4D cardiac MR images using graphics processing units. In: Biomedical Imaging: Macro to Nano, ISBI 2006. 3rd IEEE International Symposium, pp. 343-346. IEEE Computer Society Press, Los Alamitos (2006)

7. Moreland, K., Angel, E.: A fast high accuracy volume renderer for unstructured data. In: VolVis, pp. 9-16 (2004)

8. Siewerdsen, J., Antonuk, L., el-Mohri, Y., Yorkston, J., Huang, W., Cunningham, I.: Signal, noise power spectrum, and detective quantum efficiency of indirect-detection flat-panel imagers for diagnostic radiology. Medical Physics 25(5), 614-628 (1998) 